Check for updates

Cite this: Mater. Adv., 2020,

1,1613

Received 23rd March 2020

Accepted 26th July 2020

DOI: $10.1039 / \mathrm{d} 0 \mathrm{ma} 00121$

rsc.li/materials-advances

\title{
A SERS-based 3D nanobiosensor: towards cell metabolite monitoring $\dagger$
}

\author{
Sara Abalde-Cela, (D) $\ddagger^{\mathrm{a}}$ Rita Rebelo, $\ddagger^{\mathrm{bc}}$ Lei Wu, (D) $\ddagger^{\mathrm{a}}$ Ana I. Barbosa, bc \\ Laura Rodríguez-Lorenzo, ${ }^{a}$ Krishna Kant, ${ }^{a}$ Rui L. Reis, ${ }^{b c}$ Vitor M. Correlo*bc and \\ Lorena Diéguez (iD *a
}

\begin{abstract}
The development of surface-enhanced Raman scattering (SERS) spectroscopy as an analytical technique has been mainly focused on improving detection limits, nanoparticle (NP) stability and reproducibility. However, a step further on the functionalities of these promising sensing platforms is needed to enable their integration as in situ biosensors able to continuously monitor the cellular microenvironment and cell communication. Herein, we developed a biocompatible SERS hybrid material, by embedding gold-based nanostructures into gellan gum "sponge-like" hydrogels. This novel material was used as a SERS substrate for biochemical detection of disease associated cell metabolites. The optical and morphological characterisation of these 3D plasmonic sensors demonstrated the efficient incorporation of gold nanostars and silver coated gold nanorods, as well as their homogeneous distribution within the hydrogel matrices. By using these 3D plasmonic polymeric matrices we were able to prove the detection of two cancer-cell-related extracellular metabolites, lactate and thiocyanate. The SERS detection of these two small molecules is not trivial and was only possible due to the extra SERS enhancement offered by both types of anisotropic NPs. Further, the use of the gellan gum scaffold to support the NPs enables the potential use of these novel SERS platforms for the in situ growth and metabolism monitoring of 3D cell models.
\end{abstract}

\section{Introduction}

Surface-enhanced Raman scattering (SERS) spectroscopy has been demonstrated in recent years to be a powerful analytical tool offering high sensitivity and selectivity, structural information and multiplexing capacity. ${ }^{1,2}$ As a consequence, the use of SERS as an analytical technique has been extended to different fields of application, including medicine and biomolecular detection of intracellular and extracellular metabolites. ${ }^{3-9}$ SERS is based on the interaction of light with the localised surface plasmon resonance of metallic nanostructures, which enhances the inherent Raman signal of the analyte under study. Physical and chemical phenomena overcome the intrinsically low Raman efficiency, which is enhanced in SERS up to 14 orders of

\footnotetext{
${ }^{a}$ Department of Life Sciences, International Iberian Nanotechnology Laboratory (INL), Avda Mestre José Veiga s/n, 4715-310, Braga, Portugal.

E-mail: lorena.dieguez@inl.int

${ }^{b} 3 B$ 's Research Group, I3Bs - Research Institute on Biomaterials, Biodegradables and Biomimetics of University of Minho, Headquarters of the European Institute of Excellence on Tissue Engineering and Regenerative Medicine, AvePark-Parque de Ciência e Tecnologia, Zona Industrial da Gandra, 4805-017 Barco, Guimarães, Portugal. E-mail: vitorcorrelo@i3bs.uminho.pt

${ }^{c}$ ICVS/3B's-PT Government Associated Laboratory, Braga, Guimarães, Portugal

$\dagger$ Electronic supplementary information (ESI) available. See DOI: 10.1039/d0ma00121j

\$ These authors contributed equally to this work.
}

magnitude. ${ }^{10,11}$ However, the challenge for the development of this promising analytical technique relies on the ability to effectively fabricate advanced SERS substrates. In SERS, the close interaction of the nanostructure and the analyte is crucial, as it is a distance dependent effect. ${ }^{12}$ Further, the presence and control of 'hot-spot' areas with extra enhancement, due to interparticle coupling electromagnetic fields, has concentrated efforts from many researchers. ${ }^{12}$ To comply with these requirements, the main technical bottlenecks of SERS to be addressed have been the low stability of aggregated colloidal metallic NPs as well as the low affinity of certain analytes for the surface of gold or silver (the most used metallic NPs in SERS). ${ }^{13}$ Nevertheless, the construction of biocompatible SERS substrates that allow in situ biosensing in cell cultures has not been a priority in the field. However, considering the current growing applications of organ-on-a-chip models it is important that SERS substrates are compatible with long term 3D cell cultures. In this sense, the intrinsic properties of hydrogels, like their extreme hydrophilicity, make them excellent candidates as substrates supporting NPs acting as SERS enhancers. ${ }^{14,15}$ Hydrogel-based biosensors are considered an advantage over conventional systems, since they can biomimic the 3D extracellular matrix, immobilizing enzymes, proteins, aptamers or metabolites within detection systems, enhancing their stability and providing the ideal conditions to 
entrap nanostructures. ${ }^{13}$ Additionally, the combination of metallic NPs with supporting hydrogels overcomes the low stability of colloidal nanostructures, offering, simultaneously, a 3D network in which 'hot-spots' can be formed and controlled on demand. ${ }^{16}$ Furthermore, if the supporting material is able to concentrate or retain the analyte, advanced capacities towards the establishment of 3D biosensing tools can be achieved. ${ }^{17,18}$ For example, You et al. used SERS hydrogel composites to overcome the limitation of sensors, like poor long term stability issues, photobleaching and low sensitivity. ${ }^{19}$ For that, the authors converted a polyelectrolyte into a SERS-sensing platform, through the incorporation of gold nanoparticles (AuNPs) capped with Raman active molecules, for $\mathrm{pH}$ and enzymatic detection. In a different approach, Manikas et al. developed a simple method of physisorption of AuNPs on poly( $N$-isopropylacrylamide) (PNIPAm) thermoresponsive hydrogels, for the fabrication of highly efficient SERS substrates. ${ }^{20}$ This method allowed control over the interparticle distance and, therefore, the modulation of the SERS affinity and enhancement, decreasing the detection limit. Nevertheless, the use of synthetic polymers as supporting scaffolds is not the best choice for the analysis of cell metabolites, which requires superior biocompatibility.

Gellan gum hydrogels are very interesting natural scaffolds, which have demonstrated excellent non-cytotoxic performance, thus being an excellent cell growth support. ${ }^{21}$ Additionally, gellan gum is a thermo-responsive material and "sponge-like", gellan gum hydrogels present complete and rapid recovery, up to $3 \mathrm{~h}$ of application, of a compressive strain, making this a smart matrix. ${ }^{22}$ Although nanoparticle (NP)/gellan gum (GG) hybrid materials ${ }^{23}$ have been applied in various areas, to the best of our knowledge there have been no reports on their application as SERS substrates. For instance, Filpo et al. fabricated a GG/titanium dioxide nanoparticle hybrid hydrogel to clean fungal contamination on paper. ${ }^{24}$ By taking advantage of the photo-catalytic activity of titanium dioxide NPs and the mechanical soft properties of the hydrogel, this hybrid material enabled the deep cleaning and disinfection of parchment, as well as the prevention of fungi regrowth.

The combination of SERS sensing ability together with the high cell viability offered by gellan gum can be used as a potential tool for in situ and real time advanced diagnostics through the detection and quantification of specific molecules. For instance, lactate and thiocyanate play an important role in several cancer processes. The accumulation of lactate is a common feature of cancer cells, and tumour metastases are promoted by lactate-induced secretion of hyaluronan by tumour-associated fibroblasts. Thus, the accumulation of lactate in solid tumours leads to disease progression, making the real-time monitoring of lactate levels extremely important. ${ }^{25,26}$ Also, thiocyanate levels have been long associated with different types of cancer. ${ }^{27,28}$

Herein, a hybrid material was developed by embedding NPs into cell adhesive gellan gum sponge-like hydrogels (GG-SLH), which was employed as a novel SERS substrate for biochemical detection of relevant cancer-associated metabolites. Two kinds of SERS-active NPs, silver coated core-shell gold nanorods
(Au@AgNRs) and gold nanostars (AuNSs), were used to test the ability of this novel material to work as a SERS substrate. First, the synthesised NPs were incorporated into GG-SLH, forming a 3D smart matrix, which was characterised by scanning electron microscopy (SEM), extinction spectrophotometry and SERS. Second, known concentrations of Raman reporters were used to evaluate the SERS performance and limit of detection of these 3D smart matrices. Finally, two cancer related extracellular cell metabolites (lactate and thiocyanate) were detected using the hybrid material to demonstrate the potential ability of these substrates for in situ biomedical analysis.

\section{Results and discussion}

GG-SLH-NP (gellan gum "sponge-like" hydrogels with nanoparticles) was produced through the incorporation of AuNSs and Au@AgNRs into GG-SLH. Fig. 1A and B show transmission electron microscopy (TEM) images of both types of NPs before incorporation in the GG-SLH, as synthesized. Fig. 1C shows the transverse and longitudinal plasmons located at 497 and $560 \mathrm{~nm}$, respectively, for the Au@AgNRs. For the AuNSs, the plasmon peaks for the core and tips were located at $567 \mathrm{~nm}$ and $830 \mathrm{~nm}$, respectively (Fig. 1C). The absorbance peaks of the synthetized NPs are according to those described in the literature for optical and SERS detection tools. ${ }^{29-31}$ In particular, AuNSs were selected due to the extra SERS enhancement offered by their tips, and the Ag-coated AuNRs were selected (Au@AgNRs) due to the better SERS enhancement of silver $v s$. gold, as previously demonstrated. ${ }^{23,32}$

In this work, in order to obtain a good dispersion and distribution of the NPs within the GG-SLH, the NPs were added in situ before the gelation reaction of the precursor hydrogels. ${ }^{15}$ In order to test the loading efficiency into the GG-SLH, as a function of the initial concentration of NPs added, the extinction spectra of the nanostructures were obtained and the

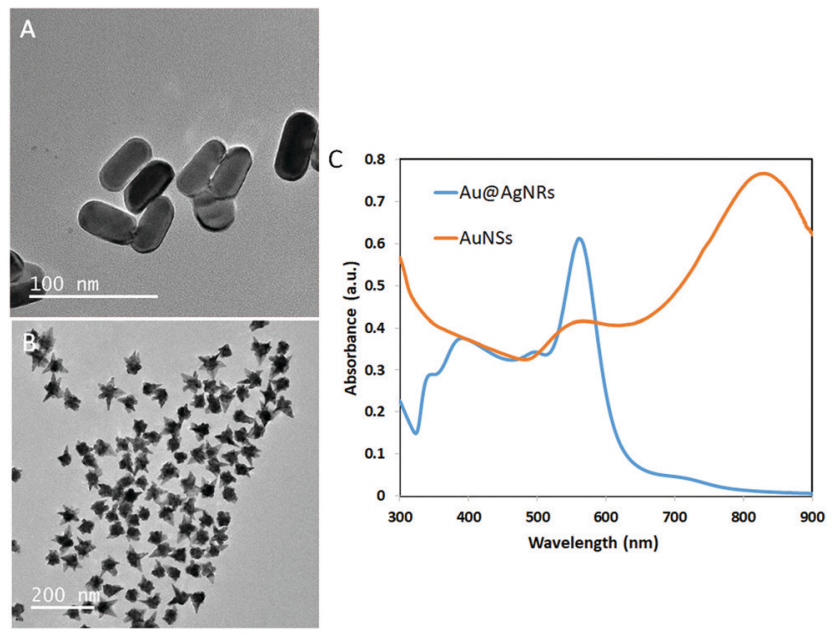

Fig. 1 (A) TEM image of Au@AgNRs as prepared before incorporation in GG-SLH; (B) TEM image of AuNSs as prepared before incorporation in GG-SLH; and (C) extinction spectra of Au@AgNRs (blue) and AuNSs (orange) normalized with the absorbance at $400 \mathrm{~nm}$. 
maximum absorbance was identified (560 nm for Au@AgNRs and $830 \mathrm{~nm}$ for AuNSs). After the NP incorporation, GG-SLHNP was dissolved in water and the absorbance of the resulting solution was measured at the specified maximum wavelengths. A calibration curve for each type of NPs was produced (Fig. S1, ESI $\dagger$ ) and used to calculate the obtained NP concentration in GG-SLH-NP. Considering the used concentration as the maximum theoretical NP concentration in GG-SLH-NP, the loading efficiency was calculated, according to eqn (1).

Loading efficiency $=[$ obtained NPs $] /[$ maximum NPs $] \times 100 \%$

As result, a loading efficiency of $64 \pm 0.005 \%$ and $95 \pm$ 0.047\% was achieved, for Au@AgNRs and AuNSs, respectively, demonstrating the successful incorporation of these nanostructures into GG-SLH. Optical images of the dry and hydrated GG-SLH and of NP loaded GG-SLH are shown in Fig. S2 (ESI†). Despite the efficient loading of NPs into the GG-SLH, the conditions that are used to prepare the hydrogel for final use may have an impact on the shape and size of the NPs, as they are subjected to stirring and high pressures and temperatures. To test the effect of gelling and freeze-drying on the morphology of the NPs, GG-SLH-NP was dissolved at the end of the process and the recovered NPs were studied to compare their initial properties with those at the end of the process. Fig. S3 (ESI $\dagger$ ) shows TEM images of the NPs recovered after collecting them from the dissolved GG-SLH-NP. TEM results confirm that both the AuNSs and the Au@AgNRs were able to stand the production conditions of the gelling and freeze-drying process. In addition, to demonstrate the successful incorporation of NPs into the matrix, these results also confirm that the morphology of the NPs was maintained after their incorporation into the GG-SLH.

Further, the arrangement of the NPs in the GG-SLH was evaluated by SEM and the results are presented in Fig. 2. Fig. 2A reveals the characteristic porous structure of the GG-SLH (Fig. 2A). The NPs, incorporated during the preparation of the precursor hydrogels, were also found in GG-SLH-NP (Fig. 2C-F). In order to have good contrast of GG-SLH-NP and the incorporated NPs, SEM images were acquired using backscattered electron mode. These micrographs show that the AuNSs (Fig. 2A, C and E) and the Au@AgNRs (Fig. 2B, D and F) were incorporated within the GG-SLH matrix in small clusters, which benefited the formation of SERS hot-spots, improving the sensitivity of the final hybrid sensor. In addition, Fig. S4 (ESI $\dagger$ ) shows the nanoCT analysis and reconstruction of the NP loaded hydrogels, in which it can be observed the inclusion of NPs and NP aggregated in the pores of the hydrogels.

Afterwards, in order to optimize the NP concentration in the GG-SLH towards achieving maximum sensor performance, two different concentrations for each of the two types of NPs were initially tested. For this purpose, the NPs were tagged with wellknown Raman reporters (RaRs) before their incorporation into the GG hydrogel precursors to report on the efficiency and homogeneity of the SERS-based embedded sensor in the 3D GG-SLH matrices.

Fig. 3A shows the SERS spectra of the GG-SLH with nonlabelled Au@AgNRs (control, in black) and with NPs labelled with 5,5-dithio-bis-(2-nitrobenzoic acid) (DTNB), at loading concentrations of $30 \mu \mathrm{M}$ (red) and $300 \mu \mathrm{M}$ (blue). For the AuNSs, the particles labelled with the reporter molecule 1-naphthalenethiol (1NAT) were used at concentrations of $500 \mu \mathrm{M}$ (red) and $900 \mu \mathrm{M}$ (blue) inside the GG-SLH (Fig. 3B). A control with non-labelled AuNSs is shown in black. The concentrations of Raman reporters on the nanoparticles were not varied.

As expected, for both types of NPs there is a very clear concentration-dependent response of the smart GG-SLH-NP reporting on the most characteristic vibrational bands for each of the RaR molecules. For DTNB and using an excitation wavelength of $633 \mathrm{~nm}$, the characteristic Raman bands are $1333 \mathrm{~cm}^{-1}$ (symmetric nitro group stretching), $1067 \mathrm{~cm}^{-1}$ (NCO stretching overlapping with aromatic ring modes), $1152 \mathrm{~cm}^{-1}(\mathrm{CH}$ deformation) and $1558 \mathrm{~cm}^{-1}$ (aromatic ring CC stretching). ${ }^{33}$
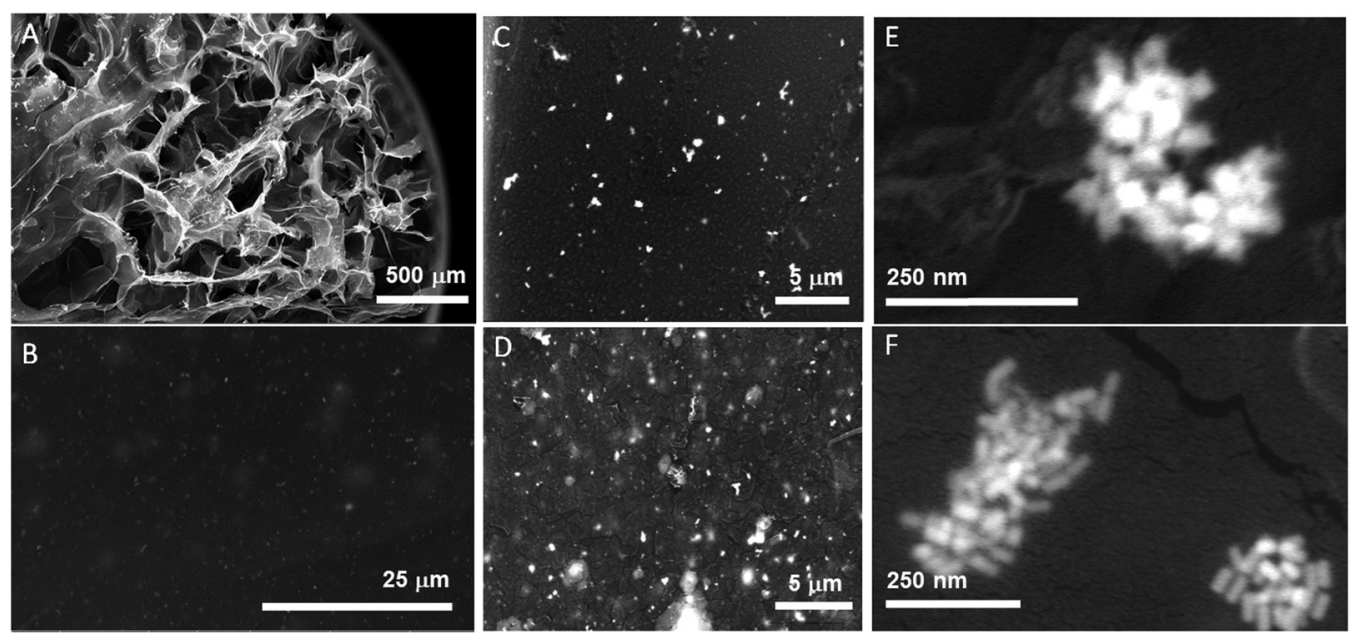

Fig. 2 SEM images of GG hydrogels at different magnifications for the AuNSs (A, C and E) and Au@AgNRs (B, D and F). 

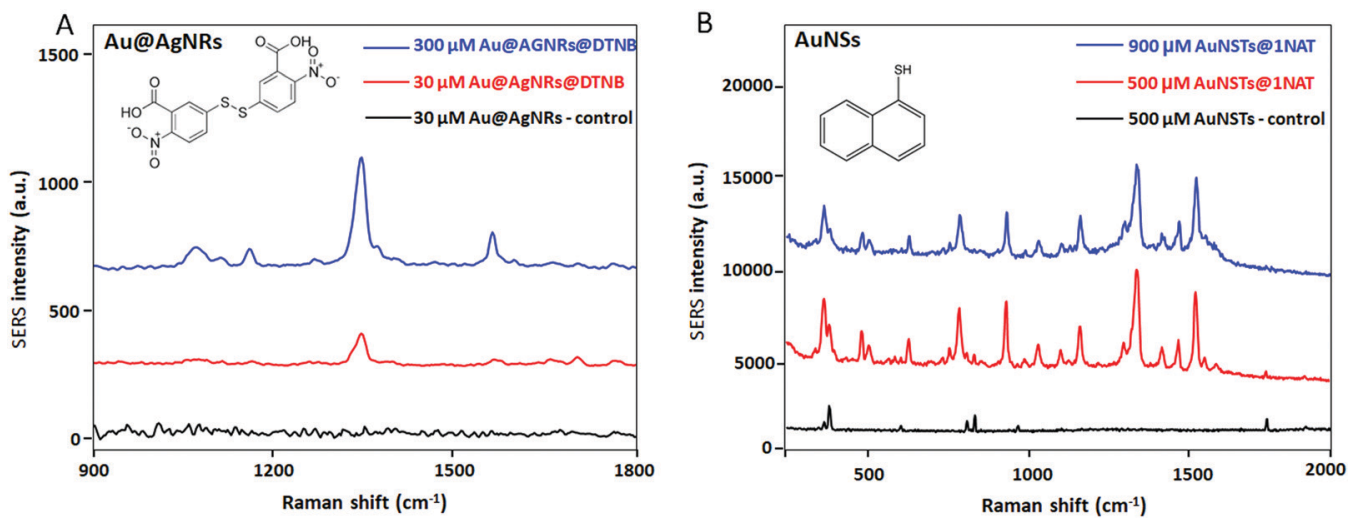

Fig. 3 SERS wet measurements of (A) GG-SLH-Au@AgNR when the Au@AgNRs were tagged with the Raman reporter DTNB (chemical structure as the inset) before NP loading in the precursor hydrogel at a concentration of NPs of $300 \mu \mathrm{M}$ (blue) and $30 \mu \mathrm{M}$ (red); and (B) GG-SLH-AuNS when the AuNSs were tagged with the Raman reporter 1NAT (chemical structure as the inset) before NP loading in the precursor hydrogel at a concentration of NPs of $900 \mu \mathrm{M}$ (blue) and $500 \mu \mathrm{M}$ (red). The black line in both (A) and (B) corresponds to the measurement of the GG-SLH loaded with both types of NPs without the Raman reporters.

For 1NAT (laser excitation $785 \mathrm{~nm}$ ) the most intense bands were those located at $389 \mathrm{~cm}^{-1}$ (CS stretching), 792, 664, 539, and $517 \mathrm{~cm}^{-1}$ (ring deformation), 968 and $822 \mathrm{~cm}^{-1}$ (ring breathing), $1197 \mathrm{~cm}^{-1}$ (CH bending) and 1553, 1503, and $1368 \mathrm{~cm}^{-1}$ (ring stretching). ${ }^{18}$ These results confirm that these types of hybrid substrates have the capacity of being efficient SERS substrates. The GG-SLH with a higher concentration of NPs offered a more intense Raman signal for each of the RaRs, as expected. For the following experiments, concentrations of $300 \mu \mathrm{M}$ and $900 \mu \mathrm{M}$ for the Au@AgNRs and AuNSs were used, respectively.

3D SERS hydrogel substrates have the capacity of acting as optical accumulators, in which molecules with low affinity for the surface of plasmonic nanoparticles can be trapped and brought into proximity to the nanostructures. In this case, the demonstration of the sensing performance of these new GG-SLH-NP SERS substrates that combine GG with anisotropic NPs was evaluated when exposed to buffers that contain RaRs at decreasing concentrations (Fig. 4).

Here, the same protocol for the incorporation of NPs into the GG-SLH was followed. However, in this case, the NPs were not previously labelled with RaRs, but were loaded bare, as synthesised, into the hydrogel. After the freeze-drying process, the produced hybrid material GG-SLH-NP $(7 \mathrm{~mm}$ diameter $\times$ $5 \mathrm{~mm}$ long cylinder) was immersed in solutions of the RaRs at different concentrations $(100 \mu \mathrm{M}-100 \mathrm{nM})$. In this way, the GG-SLH-NP matrices absorbed the molecules of interest from the solution for further analysis. Fig. 4A and B show the SERS spectra of the RaRs obtained after the analysis of GG-SLH-NP. The characteristic peaks of both DTNB (Fig. 4A) and 1NAT (Fig. 4B) were highlighted for clarity. Those peaks were detectable down to concentrations in the nanomolar range. Fig. 4C shows a comparison of the efficiency of optical accumulation of the two different types of NPs. The peak areas of $1333 \mathrm{~cm}^{-1}$ (DTNB for Au@AgNRs) and $1368 \mathrm{~cm}^{-1}$ (1NAT for AuNSs) were integrated for the different concentrations of RaR used to be absorbed by GG-SLH-NP. Once more, the SERS efficiency obtained with the AuNSs was superior to that obtained with the Au@AgNRs due to having 3 times more AuNSs embedded in the GG-SLH when compared to the NRs. As a result, the integrated areas for the characteristic peaks of DTNB were 1 order of magnitude lower than those for 1NAT. In order to further explore the NP distribution and hot-spot formation offered by these 3D SERS sensors, a surface map was obtained for GG-SLH-AuNS (Fig. 4D). The AuNSs were selected in this case due to the higher SERS performance, which can be explained as due to the higher amount of AuNSs $(900 \mu \mathrm{M})$ being incorporated with respect to Au@AgNRs $(300 \mu \mathrm{M})$. Fig. 4D (top) shows a bright field image of the SERS substrate. The map shown in Fig. 4D (bottom) is the result of the integration of the average intensity of the $1368 \mathrm{~cm}^{-1}$ (ring stretching) band of 1NAT over the area of the bright field image. The fact of the map not being fully homogenous can be explained due to the roughness of the surface of the GG-SLH material as well as by the formation of NP aggregates within the matrix, which are responsible for the high SERS performance. The stability of these hybrid substrates over time was tested six months after the measurements presented in Fig. 4 and the SERS signal was still detectable in the same conditions as at time zero after material preparation (Fig. S5, ESI $\dagger$ ).

As a proof-of-concept to demonstrate the potential of these hybrid materials towards the monitoring of relevant extracellular cellular metabolites, lactate and thiocyanate were used as model molecules. Lactate levels are related to different diseases and physiological conditions, and its detection can be applied in different fields such as sports, food science and medicine. The continuous monitoring of lactate in the human body may also improve the diagnosis and treatment of various diaseases. $^{34}$ Lactate is a small molecule having few Raman active vibrations and thus a small Raman cross section, being considered as a Raman elusive molecule. ${ }^{10}$ Traditional methods for lactate detection rely on enzymatic or immunoassay detection reactions. However, these methods are not label-free and they require the use of secondary reagents, multistep procedures, and transduction molecules. ${ }^{35}$ Also, the readout is mostly based on 

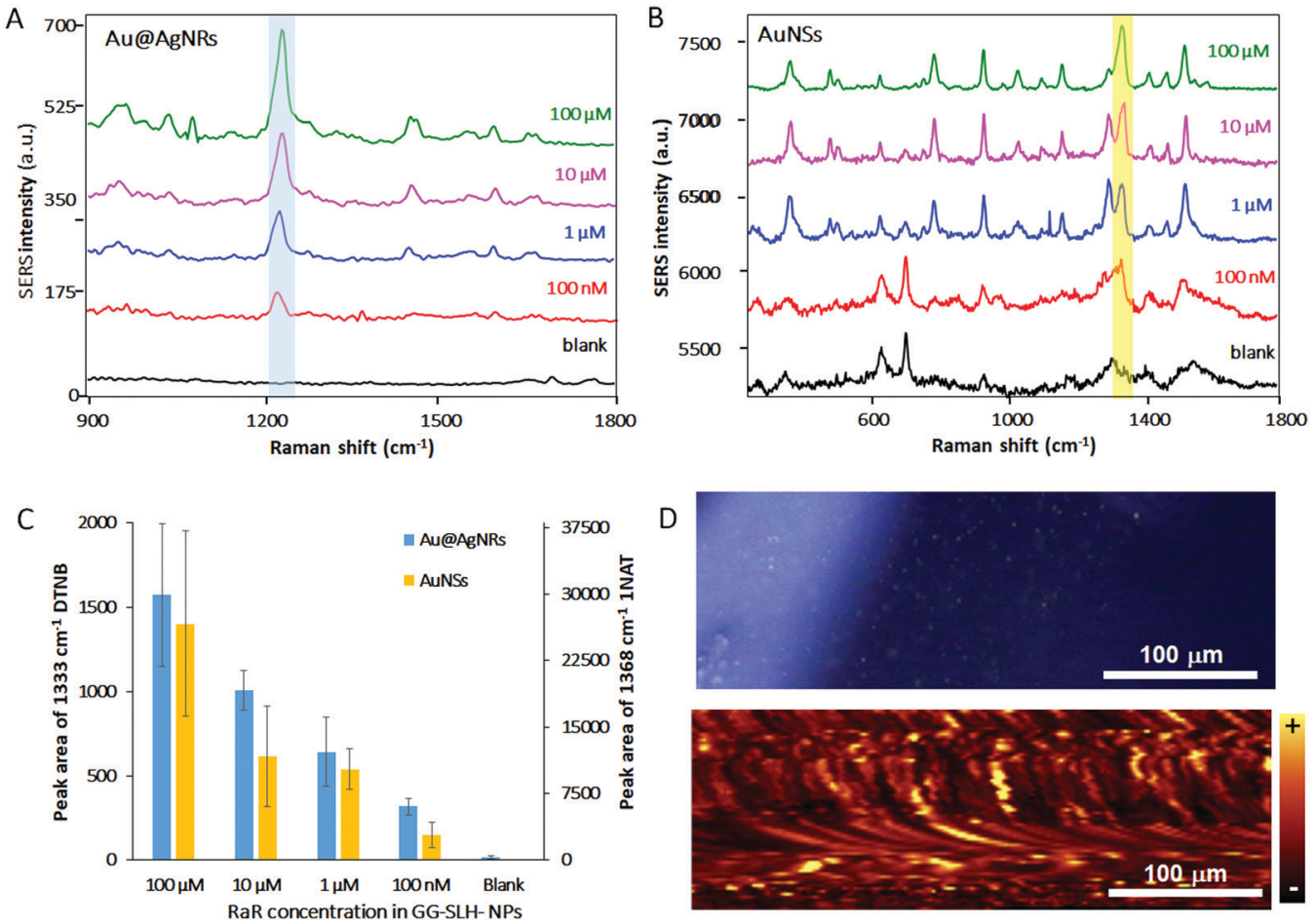

Fig. 4 SERS spectra of (A) DTNB at different concentrations captured from solution by GG-SLH-Au@AgNR; and (B) 1NAT at different concentrations captured from solution by GG-SLH-AuNS. (C) SERS semi-quantification of RaRs using both types of NPs and integrated peak area for the DTNB band (blue bars) at $1333 \mathrm{~cm}^{-1}$ (left $X$ axis) and for the 1 NAT band (yellow bars) at $1368 \mathrm{~cm}^{-1}$ (right $X$ axis). (D) Optical micrograph (top) and Raman mapping (bottom) $(400 \times 150 \mu \mathrm{m}$; step size $=2 \mu \mathrm{m} ; 10 \times$ objective; $1 \mathrm{~s}$ acquisition time $)$ of GG-SLH-AuNS after accumulation of 1 NAT from a solution of $100 \mu$ M.

electrochemistry, HPLC, fluorescence, chemiluminescence or colorimetry. ${ }^{34}$ These methods can be time consuming, difficult to automate, and not quantitative. However, by using a SERS substrate that allows one to culture cells in the same matrix in which the sensor is embedded, it would be possible to monitor lactate levels in a continuous way due to the high speed and easy automation of the reported procedure. The results of the in situ and label-free measurements of different lactate levels in the hybrid GG-SLH-AuNS matrices are shown in Fig. 5. Fig. 5A shows the bright field image of the resulting GG-SLH-AuNS $(10 \times)$ under the Raman spectrometer (top). Samples were immersed in solutions of different concentrations of lactate $(0.1,10$ and $1000 \mu \mathrm{M}$ ) and left for one hour to allow the lactate to accumulate in the GG-SLH-NP matrix. For the sample having a lactate concentration of $1000 \mu \mathrm{M}$, the area inside the white rectangle indicated in Fig. 5A was mapped (bottom) using the characteristic vibrational peak of lactate at $1424 \mathrm{~cm}^{-1}$ (symmetric stretching of $\left.\mathrm{COO}^{-}\right){ }^{36}$ It is worth highlighting that the acquisition time per

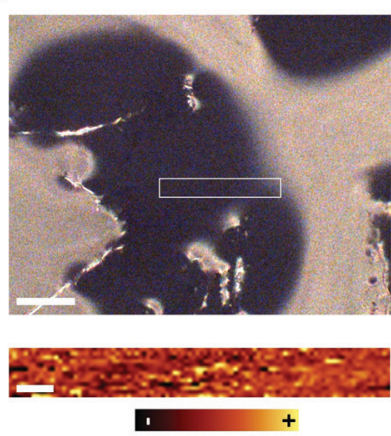

B

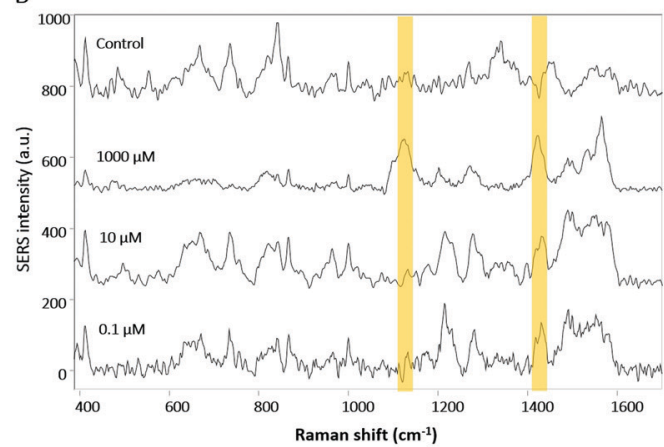

C

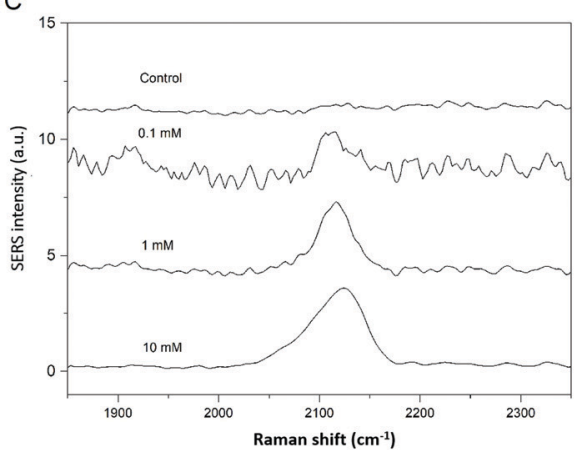

Fig. 5 (A) Top: Bright field image of GG-SLH-AuNS ( $10 \times$ objective) in which the shown area corresponds to a smaller surface section of the hydrogel (black part: pores; bright: cross-linked hydrogel); bottom: Raman mapping $(200 \times 25 \mu \mathrm{m}$; step size $=2 \mu \mathrm{m}$; acquisition time $1 \mathrm{~s}$ per spectrum) at the $1368 \mathrm{~cm}^{-1}$ band of 1NAT (scale bar bright field $=100 \mu \mathrm{m}$; scale bar map $=20 \mu \mathrm{m}$ ). (B) SERS spectra of the control (GG-SLH-AuNS without analyte) and of the lactate accumulated from lactate solutions at concentrations of 1000, 10 and $0.1 \mu \mathrm{M}$ (highlighted in yellow two characteristic peaks of lactate at $1127 \mathrm{~cm}^{-1}$ and $1420 \mathrm{~cm}^{-1}$ ). (C) SERS spectra of the control (GG-SLH-Au@AgNR without analyte) and of the thiocyanate accumulated from solutions at concentrations of 10,1 and $0.1 \mathrm{mM}$. 
spectrum $(200 \times 25 \mu \mathrm{m}$ mapping area $)$ was just one second. Despite this low acquisition time and the low Raman crosssection of the lactate molecule, the intensity of the lactate band was still recognizable using this label-free approach. The latter can only be achieved by having a 3D arrangement of hot-spots embedded within the matrices. Fig. 5B shows the average of 10 spectra for each of the tested concentrations of lactate at random points of the matrices. The bands at $1075 \mathrm{~cm}^{-1}$ (alcohol stretching $\mathrm{CO}$ ), $1127 \mathrm{~cm}^{-1}$ (rocking $\mathrm{CH}_{3}+$ alcohol stretching CO) and $1424 \mathrm{~cm}^{-1}$ (symmetric stretching of $\mathrm{COO}^{-}$) presented slight deviations from the reported Raman spectrum of lactate as expected, due to the molecule orientation and interaction with the metallic surface. ${ }^{36,37}$ However, the characteristic lactate peak at $1424 \mathrm{~cm}^{-1}$ was detectable down to $0.1 \mu \mathrm{M}$, in just 10 seconds. This acquisition time allows the continuous monitoring of lactate, and could potentially be used to predict lactate build-up in the cancer microenvironment. The lactate concentration range found in normal conditions is from 0.5 to $2.5 \mathrm{mM}$, whereas in the cancer microenvironment it can reach 7 to $8 \mathrm{mM}^{38}$ Biosensors tend to report from 10-100 $\mu \mathrm{M}$, whereas using the current approach the sensor shows a larger dynamic range from 0.1 to $1000 \mu \mathrm{M}$. By taking advantage of the GG-SLH matrix, which supports controlled 3D SERS hot-spot areas, the lactate concentration can be measured in a continuous way. Similarly, Au@AgNRs have been successfully employed to identify thiocyanate ions, another biomarker that has been commonly found in human body fluids (Fig. 5C). The characteristic Raman band at around $2115 \mathrm{~cm}^{-1}$ arising from $-\mathrm{C} \equiv \mathrm{N}$ bonds indicated the presence of thiocyanate in solution. ${ }^{39}$ For different target molecules, different GG-SLH-NP materials can be used to achieve better detection performance, according to their affinity. Furthermore, this hybrid sensor supports the culture of cells, allowing one in the future to expand into metabolite monitoring in organ-on-a-chip models.

\section{Experimental}

\section{Materials}

Unless otherwise stated all products and chemicals were purchased from Sigma-Aldrich. Milli-Q ultrapure water (Millipore, USA) was used throughout all the experiments.

\section{Synthesis of gold nanostars}

For the AuNS synthesis, a modification of the methods described by Kumar et al. was followed. ${ }^{40}$ First, for the synthesis of $15 \mathrm{~nm}$ spherical gold seeds (Au@cit), the Turkevich synthetic protocol was used. ${ }^{41}$ Briefly, $100 \mathrm{~mL}$ of $0.5 \mathrm{mM}$ aqueous solution of $\mathrm{HAuCl}_{4}$ was kept under vigorous magnetic stirring and heated at $100{ }^{\circ} \mathrm{C}$. When boiling, $5 \mathrm{~mL}$ of a $1 \%$ sodium citrate solution (previously heated to avoid thermal decomposition) was added. The solution was kept under heating and stirring until the colour turned from light yellow to dark red. Following this, the flask was left to reach room temperature and stored at $8{ }^{\circ} \mathrm{C}$ until further use. For the coating of the $\mathrm{Au}$ @cit nanoparticles with PVP (polyvinylpyrrolidone), a ratio of 60 molecules of PVP per $\mathrm{nm}^{2}$ was used. Surface calculations were performed by using the $\mathrm{ABS}_{400}$ of the $\mathrm{Au} @$ cit solution and the Lambert-Beer law. Briefly, $1 \mathrm{~mL}$ of a $1.6 \mathrm{~g} \mathrm{~mL}^{-1} \mathrm{PVP}-10 \mathrm{~K}$ solution was added to $25 \mathrm{~mL}$ of Au@cit (0.5 mM) and left under magnetic stirring overnight. The resulting solution was centrifuged at $7000 \mathrm{rpm}$ for $90 \mathrm{~min}$, and the supernatant was removed and washed under the same conditions until it was transparent. All resulting Au@PVP precipitates were re-dispersed in $25 \mathrm{~mL}$ of ethanol and stored at room temperature and under light protection until further use. Second, for the growth of the pre-formed Au@cit seeds into star-shaped nanoparticles, a $10 \mathrm{mM}$ solution of PVP $10 \mathrm{~K}\left(M_{\mathrm{W}}=10000 \mathrm{~g} \mathrm{~mol}^{-1}\right)$ was prepared in $250 \mathrm{~mL}$ of DMF ( $N, N$-dimethylformamide) and sonicated until complete dilution of PVP. Under vigorous magnetic stirring, $\mathrm{HAuCl}_{4}$ solution was added at room temperature to the DMF/ PVP solution to reach a final concentration of $0.5 \mathrm{mM}$. After 2 min, Au@PVP was added to reach a final concentration of $2.5 \times 10^{-5} \mathrm{M}$ in a total $250 \mathrm{~mL}$ reaction volume. The reaction was kept under magnetic stirring for 4 hours, followed by 3 centrifugation cycles at $4500 \mathrm{rpm}$ for $30 \mathrm{~min}$ and re-dispersion in isopropanol.

\section{Synthesis of silver-coated gold nanorods}

First, gold nanorods were fabricated using a previously reported method. ${ }^{42}$ The prepared gold nanorods were washed by centrifugation at $9000 \mathrm{rpm}$ for $20 \mathrm{~min}$ and then dispersed in water. Afterwards, $0.364 \mathrm{~g}$ of CTAB (cetrimonium bromide) was added to the purified gold nanorods, followed by the addition of $20 \mathrm{~mL}$ water, $650 \mathrm{~mL}$ of $0.1 \mathrm{M} \mathrm{AA}$ (ascorbic acid), $700 \mu \mathrm{L}$ of $15 \mathrm{mM} \mathrm{AgNO}$, and $1.2 \mathrm{~mL}$ of $0.1 \mathrm{M} \mathrm{NaOH}$ under vigorous stirring. After a few minutes, the colour gradually turned from purple to violet, green and red. Finally, the obtained nanorods were purified at $7500 \mathrm{rpm}$ for $20 \mathrm{~min}$ and then dispersed in $3 \mathrm{~mL}$ of water.

\section{Nanoparticle functionalization with Raman reporters}

AuNSs were conjugated to Raman reporter molecules, 1NAT. For this, $1 \mathrm{~mL}$ of AuNSs were mixed with different amounts of a stock solution of 1 NAT $\left(10^{-3} \mathrm{M}\right)$ depending on the target concentrations defined later in this section, and left in an orbital shaker for $2 \mathrm{~h}$. After, the resulting solution was centrifuged at $4500 \mathrm{rpm}$ for $30 \mathrm{~min}$ to remove the unbound 1NAT molecules.

Au@AgNRs were labelled with Raman reporter molecules, DTNB. For this, $1 \mathrm{~mL}$ of the prepared Au@AgNRs were mixed with $10 \mu \mathrm{L}$ of $10 \mathrm{mM}$ DTNB. After overnight reaction, the nanorods were purified at $7500 \mathrm{rpm}$ for $20 \mathrm{~min}$ to remove unbound DTNB molecules.

\section{Preparation of gellan gum "sponge-like" hydrogels loaded with nanoparticles}

GG-SLH loaded with AuNSs and Au@AgNRs were prepared using a method previously described ${ }^{15,21}$ with small modifications. Briefly, gellan powder (Sigma, USA) was dissolved in 10 different beakers with deionized water at $1.25 \%(\mathrm{w} / \mathrm{v})$ under stirring at $90{ }^{\circ} \mathrm{C}$ for approximately $20 \mathrm{~min}$. For the incorporation 
of the SERS-active nanoparticles, Au@AgNRs or AuNSs, an extra step in the preparation process was added. In more detail, after GG dissolution, the temperature was reduced to $70{ }^{\circ} \mathrm{C}$, and $500 \mu \mathrm{L}$ of different concentrations of AuNSs (0.5 and $0.9 \mathrm{mM}$ ) or Au@AgNRs ( 0.3 and $0.03 \mathrm{mM})$, both with or without RaR, were added to different GG beakers. For achieving these target concentrations a solution having an initial concentration of $1.3 \mathrm{mM}$ of AuNSs was used. Aliquots of this starting solution of $1.7 \mathrm{~mL}$ and $3.1 \mathrm{~mL}$ were centrifuged, re-suspended in $500 \mu \mathrm{L}$ and added to the GG solution, to obtain the final concentrations of 0.5 and $0.9 \mathrm{mM}$, respectively. Regarding the Au@AgNRs the initial concentration was $1.5 \mathrm{mM}$ and aliquots of $0.9 \mathrm{~mL}$ and $2.5 \mathrm{~mL}$ were centrifuged, re-suspended in $500 \mu \mathrm{L}$ and added to the GG solution to obtain final concentrations of 0.03 and $0.3 \mathrm{mM}$, respectively. For NP homogenization, a solution of $0.036 \%(\mathrm{w} / \mathrm{v})$ of calcium chloride $\left(\mathrm{CaCl}_{2}\right)$ (Merck Millipore, Germany) was added to each beaker and mixed for approximately 3 minutes. The final content of each beaker was poured into a known dimension Petri dish and the temperature was reduced in order to promote GG gelation, creating GG-NP precursor hydrogels. A phosphate buffer solution (PBS) was placed on the top of the GG-NP precursor hydrogels for $1 \mathrm{~h}$. Afterwards, a $7 \mathrm{~mm}$ diameter cylinder puncher was used to cut hydrogels with $7 \times 5 \mathrm{~mm}$ geometry, and each individual GG-NP precursor hydrogel was placed in an individual well of a 48-well plate. A volume of $1 \mathrm{~mL}$ of PBS was added to each well and left for approximately 4 hours. This process was repeated 3 times. The PBS was removed, and the 48-well plates containing the GG-NP precursor hydrogels were placed at $-80{ }^{\circ} \mathrm{C}$ for $48 \mathrm{~h}$, followed by a freeze-drying process for $72 \mathrm{~h}$. At the end of the process (Fig. S6, ESI $\dagger$ ) GG-SLH-NP was obtained.

\section{Determination of the NP loading efficiency in GG-SLH-NP}

GG-SLH-NP was placed in falcon tubes with $10 \mathrm{~mL}$ of distilled water and stirred overnight. Following this, the resulting solution (since GG is soluble in water) was centrifuged. The solutions which contained 3D matrices with AuNSs were centrifuged at $4000 \mathrm{rpm}$ for 30 minutes, while the ones containing Au@AgNRs were centrifuged at $700 \mathrm{rpm}$ for 20 minutes. After washing, the resulting solutions containing NPs were re-suspended in $1 \mathrm{~mL}$ of distilled water. The maximum absorbance spectrum peak was measured for each type of NPs using a microplate reader (BioTek Instruments), calibration curves were prepared with different amounts of each type of NPs in GG-SLH-NP and samples and standards were read at the maximum absorbance peak. Sample concentrations were determined using the equation resulting from the calibration curve and the loading efficiency was calculated according to eqn (1).

\section{Optical and morphological characterization}

For the optical characterization of the AuNSs and the Au@AgNRs, UV-vis spectra were recorded using a Shimadzu UV-2550 spectrophotometer. For the morphological characterization of both types of metallic NPs, transmission electron microscopy (TEM) images were acquired at a $200 \mathrm{kV}$ acceleration voltage using a JEM-2100-HT (JEOL, Tokyo, Japan) electron microscope. TEM samples were prepared by drop casting $10 \mu \mathrm{L}$ of the sample on a $400 \mathrm{Cu}$ mesh formvar/carbon grid, and letting the drop dry at room temperature.

The dried GG-SLH sample was used for scanning electron microscopy (SEM) analysis. The GG-SLH was first sputter coated with a platinum layer $(2 \mathrm{~nm})$ prior to SEM imaging using a QUANTA 650 FEG (FEI, Oregon, US). Imaging was done with a $10-30 \mathrm{kV}$ beam line using secondary and backscattered electron mode under high vacuum conditions.

\section{Raman spectroscopy}

A 300 Alpha confocal Raman microscope (WiTEC, Ulm, Germany) was used for the Raman and SERS analysis of the different samples. For all the analyzed samples a $10 \times$ objective was used. For the analysis of the ability of GG-SLH-NP as SERS sensors having the RaR previously incorporated together with the NPs, a piece of GG-SLH-NP (both for AuNSs and Au@AgNRs) was placed in a small container and covered with PBS $10 \mathrm{mM}$ to proceed with the SERS measurements. For the analysis of GG-SLH-Au@AgNR a $633 \mathrm{~nm}$ laser line with a grating of $600 \mathrm{~g} \mathrm{~mm}^{-1}$ was used. For GG-SLH-AuNS a $785 \mathrm{~nm}$ laser line and $1800 \mathrm{~g} \mathrm{~mm}^{-1}$ were used. The power at the sample for the GG-SLH-Au@AgNR samples and acquisition time were $5 \mathrm{~mW}$ and $2 \mathrm{~s}$, respectively. The power at the sample for the GG-SLH-AuNS samples and acquisition time were $55 \mathrm{~mW}$ and $10 \mathrm{~s}$, respectively. For the analysis of molecule uptake by GG-SLH-NP, the batches that did not have the RaR pre-loaded were immersed into $1 \mathrm{~mL}$ of solutions of concentrations ranging from $10^{-4}-10^{-7} \mathrm{M}$ of each RaR, either DTNB for the Au@AgNRs or 1NAT for the AuNSs. The GG-SLH were left in those solutions overnight, and then the solution was changed for $1 \mathrm{~mL}$ of 10 mM PBS for washing. The resulting GG-SLH-NP@RaR was analyzed under the Raman microscope under the same conditions as the RaR pre-loaded samples, as described above. Further, a Raman map of $400 \times 150 \mu \mathrm{m}$ (step size: $2 \mu \mathrm{m}$; 15000 spectra at $1 \mathrm{~s}$ acquisition time) was obtained for GG-SLHAuNS@1NAT. For the assessment of the homogeneity of the sample, several maps were taken at different positions of the substrate by placing the whole GG-SLH-NP hybrid material under the Raman system immersed in PBS. For the detection of relevant concentrations of lactate (sodium L-lactate), GG-SLH-NP samples were prepared like the ones used for the characterization of the materials with the Raman reporters; however the 3D matrices were left in the lactate solutions only for $2 \mathrm{~h}$ instead of overnight. The range of concentrations used was $10^{-5}-10^{-7} \mathrm{M}$, and the samples were then analyzed in the Raman spectrometer as before. For the single spectra acquisitions, 10 single measurements at different regions of GG-SLH-NP were taken ( $5 \mathrm{~s}$ acquisition time) for each of the samples having different concentrations of lactate. Finally, a SERS map was acquired $(200 \times 26 \mu \mathrm{m}$ step size: $2 \mu \mathrm{m}$; 1300 spectra at $1 \mathrm{~s}$ acquisition time) for the $10^{-3} \mathrm{M}$ sample.

\section{Conclusions}

In conclusion, a novel hybrid 3D biosensor was developed by combining hydrogel-based biomaterials and plasmonic nanoparticles. 
We have demonstrated the efficient incorporation of two different types of anisotropic metallic nanostructures, AuNSs and Au@AgNRs, during the GG-SLH fabrication process. Remarkably, the fabrication conditions of the 3D polymeric matrices did not have an impact on the morphology of the NPs, nor on their optical performance, as demonstrated by electron microscopy, UV-vis and Raman measurements. Furthermore, these smart 3D matrices can act as optical accumulators, trapping or absorbing molecules from external media to bring them close to the NPs acting as sensing elements in the whole hybrid material. The application of these smart 3D GG-SLH plasmonic biosensors was demonstrated by detecting lactate, a Raman elusive molecule dysregulated in the cancer microenvironment, at low acquisition times, as well as thiocyanate. As a future prospect, these $3 \mathrm{D}$ biosensors have the potential to be used as multifunctional matrices for cell growth and continuous multiplex monitoring of the cell metabolism in organ-on-a-chip models.

\section{Conflicts of interest}

There are no conflicts to declare.

\section{Acknowledgements}

The authors would like to acknowledge FROnTHERA (NORTE01-0145-FEDER-0000232) and Zeiss for nanoCT analysis, Sara Abalde-Cela acknowledges the NanoTrain4Growth II postdoctoral program funded by the E.U. Framework Program for Research and Innovation H2020 COFUND (Grant Agreement 713640), Rita Rebelo acknowledges the BREAST-IT PTDC/BTMORG/28168/2017 project.

\section{Notes and references}

1 S. Abalde-Cela, S. Carregal-Romero, J. P. Coelho and A. Guerrero-Martínez, Recent progress on colloidal metal nanoparticles as signal enhancers in nanosensing, Adv. Colloid Interface Sci., 2016, 233, 255-270.

2 K. Kant, S. Abalde-Cela, K. Kant and S. Abalde-Cela, SurfaceEnhanced Raman Scattering Spectroscopy and Microfluidics: Towards Ultrasensitive Label-Free Sensing, Biosensors, 2018, 8, 62.

3 B. Sharma, R. R. Frontiera, A.-I. Henry, E. Ringe and R. P. Van Duyne, SERS: materials, applications, and the future, Mater. Today, 2012, 15, 16-25.

4 M. F. Cardinal, et al., Expanding applications of SERS through versatile nanomaterials engineering, Chem. Soc. Rev., 2017, 46, 3886-3903.

5 P. C. Wuytens, A. Z. Subramanian, W. H. De Vos, A. G. Skirtach and R. Baets, Gold nanodome-patterned microchips for intracellular surface-enhanced Raman spectroscopy, Analyst, 2015, 140, 8080-8087.

6 J. Taylor, J. Milton, M. Willett, J. Wingfield and S. Mahajan, What do we actually see in intracellular SERS? Investigating nanosensor-induced variation, Faraday Discuss., 2017, 205, 409-428.

7 M. Ardini, et al., Live Intracellular Biorthogonal Imaging by Surface Enhanced Raman Spectroscopy using Alkyne-Silver Nanoparticles Clusters, Sci. Rep., 2018, 8, 12652.

$8 \mathrm{~V}$. Shalabaeva, et al., Time resolved and label free monitoring of extracellular metabolites by surface enhanced Raman spectroscopy, PLoS One, 2017, 12, e0175581.

9 F. Lussier, D. Missirlis, J. P. Spatz and J. F. Masson, Machine-Learning-Driven Surface-Enhanced Raman Scattering Optophysiology Reveals Multiplexed Metabolite Gradients Near Cells, ACS Nano, 2019, 13, 1409-1411.

10 R. Aroca, Surface-enhanced vibrational spectroscopy, John Wiley \& Sons, Ltd, 2006.

11 K. Kneipp, et al., Single molecule detection using surfaceenhanced Raman scattering (SERS), Phys. Rev. Lett., 1997, 78, 1667-1670.

12 G. J. Kovacs, R. O. Loutfy, P. S. Vincett, C. Jennings and R. Aroca, Distance dependence of SERS enhancement factor from Langmuir-Blodgett monolayers on metal island films: evidence for the electromagnetic mechanism, Langmuir, 1986, 2, 689-694.

13 B. Sharma, et al., High-Performance SERS Substrates: Advances and Challenges, MRS Bull., 2013, 38, 615.

14 A. Sivashanmugam, R. Arun Kumar, M. Vishnu Priya, S. V. Nair and R. Jayakumar, An overview of injectable polymeric hydrogels for tissue engineering, Eur. Polym. J., 2015, 72, 543-565.

15 L. P. da Silva, et al., Engineering cell-adhesive gellan gum spongy-like hydrogels for regenerative medicine purposes, Acta Biomater., 2014, 10, 4787-4797.

16 S. Fateixa, H. I. S. Nogueira and T. Trindade, Hybrid nanostructures for SERS: materials development and chemical detection, Phys. Chem. Chem. Phys., 2015, 17, 21046-21071.

17 V. López-Puente, S. Abalde-Cela, P. C. Angelomé, R. A. Alvarez-Puebla and L. M. Liz-Marzán, Plasmonic mesoporous composites as molecular sieves for SERS detection, J. Phys. Chem. Lett., 2013, 4, 2715-2720.

18 S. Abalde-Cela, et al., Microdroplet fabrication of silveragarose nanocomposite beads for SERS optical accumulation, Soft Matter, 2011, 7, 1321-1325.

19 Y. You, A. Nagaraja, A. Biswas, H. Marks, G. L. Coté and M. J. McShane, SERS-based hydrogel sensors for $\mathrm{pH}$ and enzymatic substrates, 2015 IEEE SENSORS, Busan, 2015, pp. 1-4, DOI: 10.1109/ICSENS.2015.7370494.

20 A. C. Manikas, G. Romeo, A. Papa and P. A. Netti, Highly Efficient Surface-Enhanced Raman Scattering Substrate Formulation by Self-Assembled Gold Nanoparticles Physisorbed on Poly( $N$-isopropylacrylamide) Thermoresponsive Hydrogels, J. Am. Chem. Soc., 2014, 30, 3869-3875.

$21 \mathrm{~J}$. T. Oliveira, et al., Gellan gum: a new biomaterial for cartilage tissue engineering applications, J. Biomed. Mater. Res., Part A, 2009, 9999, 852-863.

22 F. V. Berti, et al., Synthesis and Characterization of Electroactive Gellan Gum Spongy-Like Hydrogels for Skeletal Muscle Tissue Engineering Applications, Tissue Eng., Part A, 2017, 23, 968-979. 
23 A. Teixeira, et al., Multifuntional Gold Nanoparticles for the SERS Detection of Pathogens Combined with a LAMP-inMicrodroplets Approach, Materials, 2020, 13, 1934.

24 G. De Filpo, et al., Gellan gum/titanium dioxide nanoparticle hybrid hydrogels for the cleaning and disinfection of parchment, Int. Biodeterior. Biodegrad., 2015, 103, 51-58.

25 I. San-Millán and G. A. Brooks, Reexamining cancer metabolism: lactate production for carcinogenesis could be the purpose and explanation of the Warburg effect, Carcinogenesis, 2017, 38, 119-133.

26 F. Hirschhaeuser, U. G. A. Sattler and W. Mueller-Klieser, Lactate: A Metabolic Key Player in Cancer, Cancer Res., 2011, 71, 6921-6925.

$27 \mathrm{H}$. Wang, et al., Serum thiocyanate concentration as an indicator of smoking in relation to deaths from cancer, Environ. Health Prev. Med., 2001, 6, 88-91.

28 I. Shiue, Urinary thiocyanate concentrations are associated with adult cancer and lung problems: US NHANES, 20092012, Environ. Sci. Pollut. Res., 2015, 22, 5952-5960.

29 Z. Liang, J. Sun, Y. Jiang, L. Jiang and X. Chen, Plasmonic Enhanced Optoelectronic Devices, Plasmonics, 2014, 9, 859-866.

30 D. Fixler, R. Ankri, A. Tarnok and S. Melzer, Detection of gold nanorods uptake by macrophages using scattering analyses combined with diffusion reflection measurements as a potential tool for in vivo atherosclerosis tracking, Int. J. Nanomed., 2015, 10, 4437.

31 A. S. D. S. Indrasekara, et al., Gold nanostar substrates for SERS-based chemical sensing in the femtomolar regime, Nanoscale, 2014, 6, 8891-8899.

$32 \mathrm{~L}$. Wu, et al., A SERS-based immunoassay with highly increased sensitivity using gold/silver core-shell nanorods, Biosens. Bioelectron., 2012, 38, 94-99.

33 D. S. Grubisha, R. J. Lipert, H.-Y. Park, J. Driskell and M. D. Porter, Femtomolar Detection of Prostate-Specific
Antigen: An Immunoassay Based on Surface-Enhanced Raman Scattering and Immunogold Labels, Anal. Chem., 2003, 75, 5936-5943.

34 K. Rathee, V. Dhull, R. Dhull and S. Singh, Biosensors based on electrochemical lactate detection: a comprehensive review, Biochem. Biophys. Reports, 2016, 5, 35-54.

35 R. Martí, et al., Determination of D-lactate by enzymatic methods in biological fluids: study of interferences, Clin. Chem., 1997, 43, 1010-1015.

36 G. Cassanas, M. Morssli, E. Fabrègue and L. Bardet, Vibrational spectra of lactic acid and lactates, J. Raman Spectrosc., 1991, 22, 409-413.

37 N. C. Shah, O. Lyandres, J. T. Walsh, M. R. Glucksberg and R. P. Van Duyne, The physiological basis of physical education and athletics, Physiology: Energy, Nutrition and Human Performance, 1996, vol. 22, p. 6927.

38 N. P. Sardesai, M. Ganesana, A. Karimi, J. C. Leiter and S. Andreescu, Platinum-Doped Ceria Based Biosensor for in Vitro and in Vivo Monitoring of Lactate during Hypoxia, Anal. Chem., 2015, 87, 2996-3003.

$39 \mathrm{M}$. Bron and R. Holze, The adsorption of thiocyanate ions at gold electrodes from an alkaline electrolyte solution: a combined in situ infrared and Raman spectroscopic study, Electrochim. Acta, 1999, 45, 1121-1126.

40 P. Senthil Kumar, I. Pastoriza-Santos, B. RodríguezGonzález, F. Javier García de Abajo and L. M. Liz-Marzán, High-yield synthesis and optical response of gold nanostars, Nanotechnology, 2008, 19, 015606.

41 J. Turkevich, P. C. Stevenson and J. Hillier, A study of the nucleation and growth processes in the synthesis of colloidal gold, Discuss. Faraday Soc., 1951, 11, 55-75.

42 B. Nikoobakht and M. A. El-Sayed, Preparation and growth mechanism of gold nanorods (NRs) using seed-mediated growth method, Chem. Mater., 2003, 15, 1957-1962. 